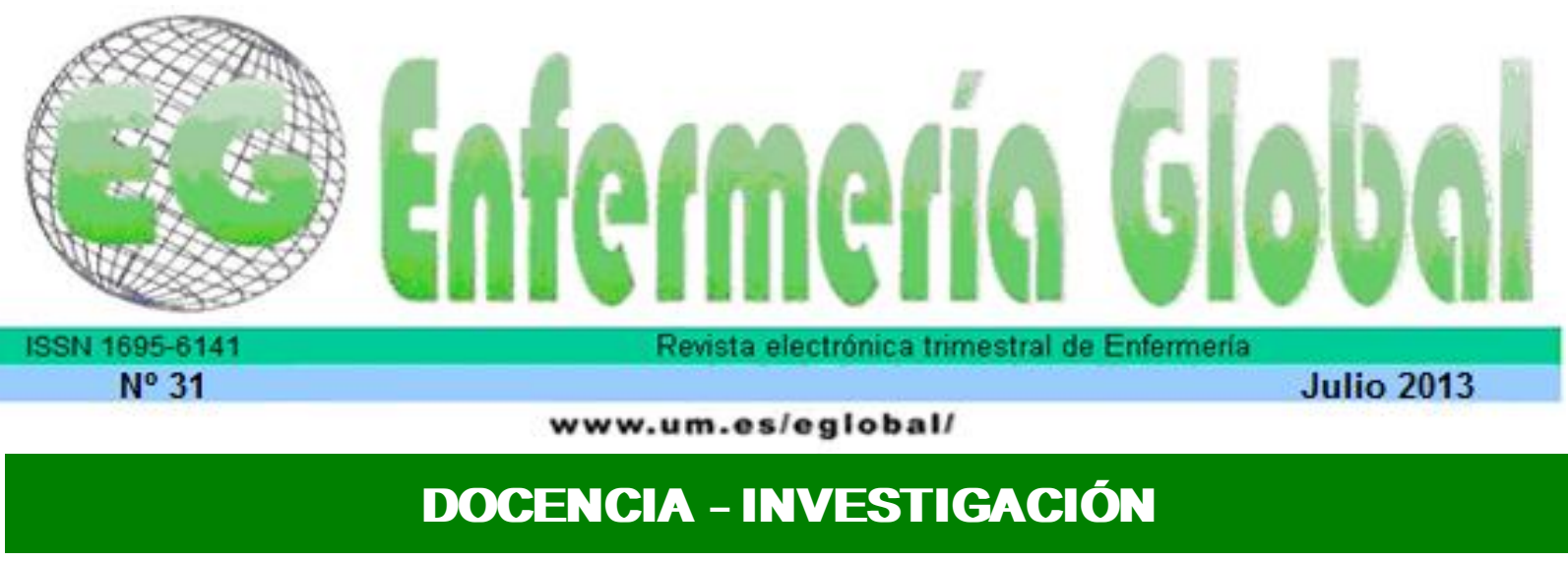

\title{
Atención en una unidad básica de salud: estudio con foco en la salud sexual de adolescentes del municipio de Rio Grande/RS
}

Atendimento em uma unidade básica de saúde: estudo com foco na saúde sexual de adolescentes do município de Rio Grande/RS

Attendance in a basic health unit: study with focus on the sexual health of adolescents from the county of Rio Grande/RS

\section{*De Oliveira Gomes, Vera Lúcia ** Ferreira Acosta, Daniele ***Silva, Camila Daiane ${ }^{* * *}$ Martins Pinheiro, Taimara ${ }^{* * *}$ Silva Souza, Catharine}

\begin{abstract}
*Doctora en Enfermería. Profesora Titular de Escuela de Enfermería. Líder del Grupo de Estudios e Investigación sobre Enfermería, Gênero ý Sociedad/GEPEGS. E-mail: vlogomes@terra.com.br **Enfermera. Mestranda do Programa de Pos-Graduación en Enfermería ***Estudiante del 9 semestre del Curso de Enfermería de la Escuela de Enfermería. Bolsista del Programa de Educación Tutorial en Enfermería ${ }^{* * *}$ Estudiante del $6^{\circ}$ semestre del Curso de Enfermería de la Escuela de Enfermería. Bolsista del Programa de Educación Tutorial en Enfermería. Universidad Federal de Rio Grande-FURG. Brasil.
\end{abstract}

Palabras clave: Salud del adolescente; Salud sexual y reproductiva, Género; Educación en enfermería. Palavras-chave: Saúde do adolescente; Saúde sexual e reprodutiva; Gênero; Educação em enfermagem.

Keywords: Adolescent health; Sexual and reproductive health; Gender; Education Nursing.

\section{RESUMEN}

Se objetivó en este estudio documental, exploratorio descrpitivo, cuantificar la atención a adolescentes y conocer los motivos que los llevan a procurar atención en una UBSF, en el municipio do Rio Grande. Todos los formularios de atención a personas con edad entre 10 y 19 años incompletos, ocurridos entre 2007 y 2010, fueron incluídos en la investigación. Los datos fueron cogidos directamente de los formularios del SIA/SUS entre octubre de 2010 y marzo de 2011. Para la tabulación, fueron introducidos en hojas del Aplicativo Microsoft Excel®. El proyecto fue aprobado por el CEPAS de la Universidad Federal do Rio Grande. Las 1532 consultas realizadas a adolescentes fueron agrupadas en cinco categorías: prevención de gravidez y de ISTs; diagnóstico de gravidez, pre-natal, aborto y puerpério; diagnóstico y tratamiento de ISTs y cáncer de cuello uterino; otros problemas ginecológicos; otros motivos. Enfocándose especificamente la salud sexual y reproductiva se contabilizaron 312 atenciones, siendo 306 para adolescentes del sexo femenino y solo seis para el sexo masculino. Aunque hay iniciación sexual precoz, son escasas las medidas relacionadas con las intervenciones 
preventivas en el grupo de edad de 10 a 14 años. Se concluye que es elevado el número de asistencias a adolescentes, sin embargo todavía prevalece el foco curativista, en que los jóvenes van a la unidad en busca de exámenes y para tratamiento de problemas. Ciertamente, promover la salud sexual de adolescentes continúa representando un desafío para los profesionales. La acogida y vínculo son indispensables para que reconozcan la unidad como un lugar propio para la discusión de sus anhelos y busca de atención a nivel preventivo.

\section{RESUMO}

Objetivou-se neste estudo documental, exploratório descritivo, quantificar 0 atendimento a adolescentes e conhecer os motivos que os levam a procurar atendimento em uma UBSF, no município do Rio Grande. Todos os formulários de atendimento a pessoas com idade entre 10 e 19 anos incompleto, ocorridos entre 2007 e 2010, foram incluídos na pesquisa. Os dados foram colhidos diretamente dos formulários do SIA/SUS entre outubro de 2010 e março de 2011. Para a tabulação, foram inseridos em planilhas do Aplicativo Microsoft Excelß. O projeto foi aprovado pelo CEPAS da Universidade Federal do Rio Grande. As 1532 consultas realizadas a adolescentes foram agrupadas em cinco categorias: prevenção de gravidez e de ISTs; diagnóstico de gravidez, pré-natal, aborto e puerpério; diagnóstico e tratamento de ISTs e câncer de colo uterino; outros problemas ginecológicos; outros motivos. Enfocando-se especificamente a saúde sexual e reprodutiva foram contabilizados 312 atendimentos, sendo 306 para adolescentes do sexo feminino e apenas seis para o sexo masculino. Embora haja iniciação sexual precoce, são escassas as medidas relacionadas às intervenções preventivas na faixa etária de 10 a 14 anos. Concluiu-se que é elevado o número de atendimentos a adolescentes, entretanto ainda prevalece o foco curativista, em que os jovens procuram a unidade em busca de exames e para tratamento de problemas. Certamente, promover a saúde sexual de adolescentes continua representando um desafio para os profissionais. O acolhimento e vínculo são indispensáveis para que reconheçam a unidade como um local próprio para a discussão de seus anseios e busca de atendimento em nível preventivo.

\section{ABSTRACT}

This documental, descriptive exploratory study was aimed to quantify the attendance of adolescent people and to know their reasons to look for treatment in a UBSF, in the county of Rio Grande. All of the attendance forms of people with age between 10 and 19 years incomplete, occurred from 2007 to 2010 , were included in the research. The data were collected directly from the SIA/SUS forms between October of 2010 and Marcho f 2011.To the tabulation, were inserted in Microsoft Excel® spreadsheets. The Project was approved by the Federal University of Rio Grande (FURG) CEPAS. The 1532 consultations in adolescents were combined in five categories: pregnancy and ISTs prevention; pregnancy diagnosis; prenatal, abortion and puerperium; diagnosis and treatment of ISTs and cancer of uterine cervix; other gynecological problems; other reasons. Focusing specifically in sexual and reproductive health were counted 312 attendance, being 306 female adolescents and Just six to the opposite sex. Despite the early sexual initiation, there are few measures related to preventive interventions in the age group of 10 to 14 years. Was concluded that the number of attendances to adolescents is elevated, however still prevails curativistic focus, in which the youth go to the unit looking for exams and treatment for their health issues. In fact, promoting the sexual health to adolescents still represents a challenge for the professionals. The reception and bond are essential to make them recognize the unit as a proper local to discuss their longings and search the understanding in preventive level.

\section{INTRODUCCIÓN}

La adolescencia es una etapa fundamental del desarrollo humano, caracterizada por grandes resultados, desafíos, vivencias y expectativas ${ }^{(1)}$. En este periodo ocurren grandes transformaciones y adquisiciones, tanto en la esfera física como en la cognitiva y psicosocial. Tales cambios se materializan de forma flexible o de manera restrictiva, de acuerdo con las reglas y culturas en que se vive ${ }^{(2)}$.

Es en esta fase cuando sucede el descubrimiento de la sexualidad, los primeros enamoramientos, relaciones, y la iniciación sexual ${ }^{(3)}$. Es normal la busca de 
autonomía de proyectos y prácticas, ejercida de forma singular y con la urgencia de la juventud ${ }^{(4)}$. Por otro lado, son muchas las situaciones en que la orientación del adolescente se limita a los aspectos anatomofisiológicos del aparato reproductor y patologías derivadas de la práctica sexual sin protección. Números contundentes demuestran esta forma reduccionista de enfocar la sexualidad. Aproximadamente $36,0 \%$ de los adolescentes tuvieron la primera relación sexual antes de los 15 años, de estos 16,0\% tuvieron más de 10 compañeros(as) sexuales en la vida y aproximadamente $7,0 \%$ tuvieron más de cinco compañeros(as) eventuales en el último año ${ }^{(5)}$.

Según estudio de la Federación Internacional de Planificación Familiar (IPPF), los índices de aborto en Brasil van en aumento entre adolescentes. En el año 2005, se contabilizaron 2.781 atenciones por complicaciones post aborto, en el grupo de edad entre 10 y 14 años y 46.504 entre adolescentes con edades de 15 a 19 años ${ }^{(6)}$. En lo que se refiere a las gestaciones, anualmente en Brasil se registran cerca de 30.000 partos de adolescentes con edades entre 10 y 14 años ${ }^{(7)}$.

El comportamiento contraceptivo en la juventud presenta una dinámica propia que varía de acuerdo con el tipo de relación afectivo-amorosa establecida entre los compañeros $^{(8)}$. Si ésta es eventual o reciente, es común el uso de preservativos; si estable, las medidas preventivas son relegadas a un segundo plano o inclusoo abandonadas, práctica esta interpretada por los jóvenes como una prueba de amor. Esta conducta confirma la necesidad de una asistencia individualizada y multidisciplinar que abarca familia, escuela y servicios de salud en pro de una educación contextualizada y dirigida a las reales necesidades de los adolescentes.

A pesar de este nuevo contexto, padres, madres y familiares tienen dificultad en conversar con los jóvenes sobre la sexualidad como algo saludable y placentero, sea por falta de información o por restricción ${ }^{(9)}$. En este sentido, no se puede ignorar que, en muchos de ellos, se inculcó la idea de que sexo y sexualidad son asuntos feos, sucios y hasta prohibidos. Resulta curioso percibir que muchas veces son permitidas bromas picantes y hasta estimuladas en ambiente familiar, mientras que asuntos referentes a la propia sexualidad tienen espacio limitado o incluso nulo. De este modo, la discusión y la confrontación con la sexualidad de los hijos e hijas se convierten en uno de los principales dilemas de la familia del adolescente (10), constituyéndose en obstáculo para la promoción de su salud sexual y reproductiva.

El adolescente tiene el derecho a "recibir información de una forma amplia y democrática, para que pueda tomar decisiones con libertad y conocimiento" (11:3). Así, desde que es capaz de evaluar su problemática y conducirse en busca de la solución, los adolescentes tienen derecho a la confidencialidad. La quiebra del secreto profesional solo debe ocurrir en circunstancias especiales, relacionadas con su seguridad y protección ${ }^{(11)}$. En lo que se refiere a la familia, es importante que se comprenda ese derecho, pues la pérdida de la confidencialidad, sin un motivo claro, afectará la interacción adolescente y profesional de salud, dificultando o incluso inviabilizando atenciones futuras y alejando a los jóvenes de los servicios de salud.

De forma muy clara, la guía de salud del adolescente explicita que los derechos de los jóvenes precisan ser preservados durante y después de las consultas en establecimientos de salud. Este documento destaca que el secreto solo podrá ser roto con la autorización del cliente adolescente o si hubiera daños a su salud o a terceros. 
A pesar de estas recomendaciones, la frecuencia de adolescentes en los servicios de salud es baja, pues los jóvenes brasileños no consideran tales servicios como prioritarios para la obtención de informaciones confiables sobre sexualidad (4). Sumado a esto, existe la vergüenza y el miedo a ser repreendido por el profesional de salud ${ }^{(12)}$ o por familiares. Basándonos en estos datos, se realizó el presente estudio con el objetivo de cuantificar la atención a adolescentes y conocer los motivos que los llevan a procurar atención en una Unidad Básica de Salud de la Familia (UBSF).

\section{MATERIAL Y MÉTODO}

Se trata de un estudio documental, exploratorio descriptivo, con abordaje cuantitativo, realizado en una UBSF de la periferia de la ciudad do Rio Grande/RS. Esta unidad, en 2010, contaba con seis Agentes Comunitarios de Salud que atendían a 1065 familias, con aproximadamente tres mil personas, de estas 452 eran adolescentes, estando 263 en el grupo de 10 a 14 años y 189 en el grupo de 15 a 19 años incompletos. Se adoptó el concepto de la Organización Mundial de la Salud como criterio de edad definidor de la muestra. Así, todos los formularios de atención a personas con edad entre 10 y 19 años incompletos, ocurridos entre 2007 y 2010, fueron incluídos en la investigación. La colecta fue realizada directamente de los formularios del Sistema de Informaciones Ambulatorias del Sistema Único de Salud (SIA/SUS) entre octubre de 2010 y marzo de 2011. Las informaciones recogidas se limitaron a la edad, sexo y motivo de la asistencia.

Para la tabulación, los datos fueron incluidos en hojas del Aplicativo Microsoft Excel® y sometidos al análisis descriptivo. El proyecto fue aprobado por el Comité de Ética en Investigación de la Universidad Federal do Rio Grande con parecer № 81/2009.

\section{RESULTADOS}

En el periodo de 2007 a 2010 se realizaron 1532 atenciones a adolescentes en el grupo de 10 a 19 años incompletos en la UBSF en estudio. Los motivos de atención fueron agrupados en cinco categorías: a) prevención de gravidez y de Infecciones Sexualmente Transmisibles (ISTs); b) diagnóstico de gravidez, pre-natal, aborto y puerperio; c) diagnóstico y tratamiento de ISTs y cáncer de cuello uterino; d) otros problemas ginecológicos; e) otros motivos.

En la primera categoría, se incluyeron todas la atenciones referentes a orientaciones sobre el uso de contraceptivo y preservativo, busca de contraceptivos, busca de preservativos y de contracepción de emergencia, en fin, todas las consultas con objetivo de prevención de gravidez e ISTs. En la segunda categoría, se agruparon todas las consultas derivadas de sospecha de gravidez, aborto, parto y puerpério. Así, se incluyó amenorrea acompañada de mareo; test de gravidez; ausencia de movimientos fetales; gestante con sangrado vaginal; pérdida de líquido amniótico; envío de la gestante al hospital; curativos de cesárea; depresión puerperal y aborto inducido. Los motivos agrupados en la categoría de diagnóstico y tratamiento de ISTs y cáncer de cuello uterino fueron la realización de examen citopatológico, algunas veces registrado como pre-cáncer; exámen de HIV; verruga vaginal; herpes genital; lesiones vaginales y anales. En la categoría otros problemas ginecológicos, se incluyeron las consultas motivadas por metrorragia, leucorrea, nódulos, dolor o abceso en las mamas; galactorrea; hemorragia menstrual; quistes en el ovario; dispareunia; tensión pre-menstrual y pos-operatorio de ooferectomía. Finalmente, en 
la categoría otros motivos, se agruparon todas las consultas no relacionadas con la salud sexual y reproductiva.

Si tenemos en cuenta que a partir del año 2000 el IBGE incluyó el grupo de edad de 10 a 14 años entre los indicadores de fecundidad y que son escasos los datos epidemiológicos referentes a ese grupo de edad, las edades de los adolescentes se clasificaron en dos grupos: de 10 a 14 años y de 15 a 19 años incompletos.

De las 1532 asistencias registrados, 787 (51,4\%) estuvieron destinadas a adolescentes con edad entre 10 y 14 años y 745 (48,6\%) a los de edad entre 15 y 19 años incompleto. Enfocadas específicamente a la salud sexual y reproductiva se contabilizaron $312(20,36 \%)$ asistencias, siendo $306(98,7 \%)$ a adolescentes de sexo femenino y seis $(1,92 \%)$ para el sexo masculino.

Entre las atenciones prestadas a adolescentes con edad entre 10 y 14 años, solo 52 $(6,6 \%)$ acudieron con objetivos de promoción de la salud sexual reproductiva. En la edad de 10 años ocurrieron solo dos asistencias, motivadas por lesiones vaginales y ardor al orinar. Con 11 años fueron atendidos dos adolescentes y los motivos de las consultas fueron: diagnóstico de fimosis y retirada de puntos del saco escrotal. Para clientes con doce años se realizaron 15 asistencias, siendo tres para realización de pre-natal, dos para test de gravidez (BHCG) y una para diagnóstico de HIV. Entre las edades de 13 y 14 años, totalizaron 33 asistencias, siendo 12 para prevención de gravidez e IST's, 13 distribuidos entre diagnóstico de gravidez, pre-natal y puerpério, dos para diagnóstico y tratamiento de IST's, y seis para otros problemas ginecológicos entre ellos hemorragias menstruales y secreción vaginal.

Entre los adolescentes con edad comprendida entre 15 y 19 años incompletos, se realizaron $745(48,6 \%)$ asistencias, siendo $260(34,89 \%)$ referentes a la salud sexual y reproductiva. De estas, $80(30,76 \%)$ buscaron atención para la prevención de gravidez e IST's; 127 (48,84\%) para diagnóstico de gravidez, pre-natal y puerpério; 26 (10\%) para diagnóstico y tratamiento de IST's y $27(10,38 \%)$ para otros problemas ginecológicos. A pesar de que 80 adolescentes objetivaron la prevención de gravidez o IST, destaca el que 79 acudieron al servicio de salud en busca de anticonceptivo oral o inyectable, solamente uno solicitó preservativo masculino, con miras a la doble protección. Además, se constató que el número de consultas destinadas a pre-natal $75(28,84 \%)$ atenciones, fue muy semejante al de consultas con el foco de prevención en la gravidez.

\section{DISCUSIONES}

Conforme estimación de la Organización de las Naciones Unidas (ONU), los adolescentes representan cerca del $25 \%$ de la población mundial. Al sufrir intensos cambios biopsicosociales, su singularidad les transforma en grupo vulnerable para ciertos problemas de salud ${ }^{(13)}$. La existencia de servicios de salud de calidad así como de profesionales capacitados para atender adolescentes se torna un desafío para alcanzar mejores condiciones de vida y de salud de los adolescentes.

Al igual que en este estudio, otras investigaciones divulgaron que los adolescentes utilizan poco el servicio de salud ${ }^{(14)} \mathrm{y}$, cuando lo hacen, la mayor parte de la asistencia se centra solo en la enfermedad ${ }^{(15)}$, a través de consultas y exámenes, según lo propuesto por la Estrategia de Salud de la Familia. La búsqueda de intervenciones preventivas, como métodos contraceptivos, solamente ocurrió a partir 
de los 13 años. Sumado a esto, de las 15 consultas realizadas a los adolescentes con 12 años de edad, todas evidenciaron la iniciación sexual precoz y sin protección.

En 2004 apareció un estudio que incluía a 16.400 adolescentes de las capitales brasileñas que mostraba que la primera relación sexual, entre los chicos, fue a los 14,5 años y entre las chicas a los 15,5. La misma investigación divulgó que una de cada diez estudiantes queda embarazada antes de los 15 años ${ }^{(16)}$. Esta es una realidad visible, conocida por todos, y que confirma la necesidad de la intensificación de acciones promotoras de la salud sexual y reproductiva desde el inicio de la adolescencia.

Otra característica típica de esta fase es la "relación" definida como enamoramiento sin compromiso ${ }^{(17)}$. En este aspecto, los chicos están más expuestos, pues es común entre ellos competiciones e iniciación sexual precoz con el objetivo de mostrar su masculinidad ${ }^{(18)}$. Sin embargo, en lo que se refiere a la salud sexual y reproductiva, de las $312(20,36 \%)$ asistencias, solo $6(1,92 \%)$ se prestaron a jovenes del sexo masculino corroborando la constatación de que desde temprano los hombres acuden menos a los servicios de salud ${ }^{(19)}$. Tal hecho involucra factores socioculturales y creencias de que el hombre no enferma, y que la enfermedad es considerada como una señal de fragilidad, o incluso que los servicios de salud están destinados al niño, a la mujer y al anciano ${ }^{(20)}$.

En cuanto a los adolescentes en el grupo de edad entre 15 y 19 años se supone que con la proximidad de la transición a la fase adulta adquieren una mayor autonomía e independencia en diversos campos de la vida, incluyendo su sexualidad ${ }^{(16)}$. Esto se comprueba en la relación positiva entre mayores grupos de edad y el uso de métodos contraceptivos como píldora ${ }^{(21)}$ y el preservativo masculino en ambos sexos ${ }^{(22)}$.

Sin embargo, esta independencia no disminuye el riesgo de exposición a la gravidez e ISTs. Es preciso un trabajo de base, multidisciplinar, siendo el servicio de salud responsable de la interacción entre las familias y la escuela. Los padres acostumbran transferir el papel educativo a terceros, pues temen conversar sobre este asunto, por eso, el diálogo interfamiliar representa un factor de protección para los adolescentes $^{(22)}$.

La actuación profesional junto a adolescentes precisa estar libre de preconceptos, juicios y basada en el respeto, en la libertad de expresión de los sentimientos, miedos, y dudas. Los jóvenes deben sentirse acogidos por todo el equipo para que la Unidad de Salud sea percibida como una referencia para la promoción de la salud.

Se destaca también la necesidad de implementar programas específicos para esta población, con profesionales capacitados y sensibles a la problemática juvenil, que desarrollen actividades y enfoquen asuntos sugeridos por ellos. La atención inadecuada o incluso la no atención a adolescentes se justifica muchas veces por la falta de recursos educativos y estructura inadecuada ${ }^{(15)}$, por eso cabe enfatizar que, la mayoría de las veces, los jóvenes precisan ser oidos de forma atenta y respetuosa, para que se cree un enlace, y ellos retornen a los servicios de salud.

En ese sentido, ninguna oportunidad puede ser desperdiciada y cualquier atención dirigida a adolescentes debe ser vista como um momento propicio para la promoción de la salud sexual y reproductiva, así como de diagnóstico y tratamiento de problemas. 


\section{CONCLUSIONES}

Los resultados del presente estudio reflejan que el número de adolescentes que busca atención en la UBSF es elevado, de la misma forma que son diversos los motivos para esa ayuda. Sin embargo, cuando el foco es la promoción de la salud sexual y reproductiva, se percibe que el número de asistencias es insuficiente para que tal objetivo sea alcanzado. Con ello se pierden excelentes oportunidades de prestar atención individualizada y colectiva a esta población para proporcionarles una adolescencia saludable.

Ciertamente, promover la salud sexual de los jóvenes continúa representando un desafío para los equipos de las Unidades Básicas de Salud de la Familia (UBSF). Es necesario invertir en la formación permanente de los profesionales de la salud, con foco en los aspectos éticos y legales que impregnan la atención en ese grupo de edad. El vínculo y la acogida son indispensables para que los jóvenes reconozcan la UBSF como un lugar propio para la discusión de sus anhelos y busca de atención a nivel preventivo.

El aflorar de la sexualidad es un proceso que sobrepasa el cuerpo biológico y las edades limítrofes. El compromiso de los profesionales de salud en este proceso, sólo será efectivo si consideran la individualidad y el momento de vida de cada adolescente. Para ello, las cuestiones presentadas por los jóvenes precisan ser problematizadas con seriedad, ética y competencia, pues la busca de alternativas debe ser planificada con ellos y no para ellos.

\section{REFERENCIAS}

1 Brasil. Ministério da Saúde. Marco Teórico e Referencial: saúde sexual e saúde reprodutiva de adolescentes e jovens. Secretaria de Atenção à saúde. Brasília: Ministério da Saúde, 2006.

2 Taquette SR. Sexualidade na adolescência. 205-212 p. In: Brasil. Ministério da Saúde. Departamento de Ações Programáticas Estratégicas: Saúde do Adolescente: competências e habilidades. Brasília: Editora do Ministério da Saúde, 2008. [acesso em 27 jul 2011]. Disponível em: http://portal.saude.gov.br/portal/arquivos/pdf/saude adolescente.pdf

3 Cruzeiro ALS, Souza LDM, Silva RA, Horta BL, Muenzer RM, Faria AD. Iniciação Sexual entre adolescentes de Pelotas, Rio Grande do Sul. Rev Bras Crescimento Desenvol Hum. 2008; 18(2): 116-125.

4 Brasil. Ministério da Saúde. Marco Teórico e Referencial: saúde sexual e saúde reprodutiva de adolescentes e jovens. Secretaria de Atenção à saúde. Brasília: Ministério da Saúde, 2007a. 56 p.

5 Brasil. Boletim epidemiológico AIDS e DST. Brasília: Ministério da Saúde, $2007 b$. [acesso em 05 abr 2010]. Disponível em: http://www.aids.gov.br/data/documents/storedDocuments

6 Silva M. Aborto é crescente entre crianças e adolescentes. Jornal Pulsar Brasil. Rio de Janeiro, 2007. [acesso em 20 out 2011]. Disponível em: http://www.brasil.agenciapulsar.org/nota.php?id=1993

7 Silva RC. Responsabilidade no exercício da sexualidade do adolescente. In: Pereira JL, Fanelli C, Pereira RC, Rios S, organizadores. Sexualidade na adolescência no novo milênio. Rio de Janeiro (RJ): UFRJ/Pró-Reitoria de Extensão, 2007. 
8 Borges ALV, Fujimori E, Hoga LAK, Contin MV. Práticas contraceptivas entre jovens universitários: o uso da anticoncepção de emergência. Cad. Saúde Pública. 2010; 26(4): 816-826.

9 Martíni JG. Educação Sexual em tempos de HIV/AIDS. Enfermería Global. 2007; 11 (noviembre): 1-17.

10 Almeida $\mathrm{ACCH}$, Centa ML. A família e a educação sexual dos filhos: implicações para a enfermagem. Acta Paul Enferm. 2009; 22(1): 71-76.

11 Ruzany MH. Atenção ao adolescente: considerações éticas e legais. 26-34p. In: Brasil. Ministério da Saúde. Departamento de Ações Programáticas Estratégicas: Saúde do Adolescente: competências e habilidades. Brasília: Editora do Ministério da Saúde, 2008. [acesso em 27 jul 2011]. Disponível em: http://portal.saude.gov.br/portal/arquivos/pdf/saude adolescente.pdf

12 Silva LN, Ranna FF. Captação e acolhimento do adolescente. In:Manual de atenção à saúde do adolescente.21-26 p. Prefeitura da cidade de São Paulo. Secretaria Municipal da Saúde. Coordenação de Desenvolvimento de Programas e Políticas de Saúde. São Paulo: SMS, 2006.

13 Reato LFN. A consulta médica. 79-83 p. In:Manual de atenção à saúde do adolescente. Prefeitura da cidade de São Paulo. Secretaria Municipal da Saúde. Coordenação de Desenvolvimento de Programas e Políticas de Saúde. São Paulo: SMS, 2006. 328p.

14 Goldbaum M, Gianini RJ, Novaes HMD, César CLG. Utilização de serviços de saúde em áreas cobertas pelo programa saúde da família (Qualis) no Município de São Paulo. Rev Saúde Pública. 2005; 39 (1): 90-99.

15 Vieira RP, Machado MFAS, Bezerra IMP, Machado CA. Assistência à saúde e demanda dos serviços na estratégia saúde da família: a visão dos adolescentes. Cogitare Enferm. 2011; 16(4): 714-720.

16 Ribeiro PCP. A Saúde Sexual e Reprodutiva. 104-37 p. In: Atenção a Saúde do adolescente. Belo Horizonte: Secretaria de Saúde do Estado de Minas Gerais, 2006.

17 Bezerra CP, Pagliuca LMF. A vivência da sexualidade por adolescentes portadoras de deficiência visual. Rev Esc Enferm USP. 2010; 44(3):578-83.

18 Gubert D, Faganello VSM. Iniciação sexual de homens adolescentes. Ciência \& Saúde Coletiva. 2009; 14: 1119 - 1128.

19 Ferrari RAP, Thomson Z, Melchior R. Atenção à saúde dos adolescentes: percepção dos médicos e enfermeiros das equipes da saúde da família. Cad. Saúde Pública, Rio de Janeiro, 2006; 22(11): 2491-2495.

20 Brasil. Ministério da Saúde. Política Nacional de Atenção Integral à Saúde do Homem. Princípios e Diretrizes. Brasília: Ministério da Saúde, 2008.

21 Figueiredo R, Alves MCGP, Escuder MM, Pupo LR.Comportamento Sexual, Uso de Preservativos e Contracepção de Emergência por Adolescentes do Município de São Paulo - estudo com estudantes de escolas públicas de Ensino Médio. São Paulo: Instituto de Saúde, 2008.

22 Teixeira AMFB, Knauth DR, Fachel JMG, Leal AF. Adolescentes e uso de preservativos: as escolhas dos jovens de três capitais brasileiras na iniciação e na última relação sexual Cad. Saúde Pública, Rio de Janeiro. 2006; 22 (7): 1385-1396.

ISSN 1695-6141

(C) COPYRIGHT Servicio de Publicaciones - Universidad de Murcia 